\title{
Two-dimensional remote interferometric stage encoder through a single access port using range-resolved interferometry
}

\author{
Kieran B. Wiseman, Thomas Kissinger* and Ralph P. Tatam \\ Engineering Photonics, Cranfield University, Cranfield, MK43 0AL, United Kingdom
}

\begin{abstract}
In this work, using our range-resolved interferometry (RRI) signal processing technique, we present a novel approach to multidimensional displacement measurements using only a single optical access port and very simple optical setup. By utilising surface reflections from a stage-mounted moving beamsplitter and two orthogonal stationary reference mirrors, two interferometers for the two Cartesian measurement directions are formed. With RRI, the interferometric phase signals of both interferometers can be independently demodulated, allowing simultaneous measurements of displacement in both dimensions using a single continuous-wave laser diode source and a single photodetector. In this paper, the capabilities of this approach are demonstrated using a proof-of-concept experiment with a multidimensional Piezoelectric stage performing a variety of stage movements. Measurements of displacements over a nominal stage working range of $\pm 50 \mu \mathrm{m}$ are presented, demonstrating independent, simultaneous displacement measurements of two dimensions. The presented measurements show nanometer-level displacement resolutions with typical noise densities of $0.02 \mathrm{~nm} / \sqrt{\mathrm{Hz}}$ over a $21 \mathrm{kHz}$ bandwidth. It is thought that this approach could offer an interesting alternative to existing interferometric techniques for multi-dimensional metrology, benefiting from both simplicity and cost-effectiveness whilst maintaining the advantages that make optical techniques attractive to scientific and industrial applications.
\end{abstract}

Keywords: Displacement Measurement, Range-Resolved Interferometry, Diode Lasers, Dimensional Metrology, Optical Encoder

\section{INTRODUCTION}

As technology advances, so does the need for high-precision and versatile metrological methods. Existing position encoders for absolute distance and relative displacement measurements include both optical and non-optical techniques ${ }^{1}$. Non-optical techniques such as Hall-effect and capacitive sensors often require close contact with the target object in order to operate, a restriction that adds complication in many applications. Optical techniques can be broadly separated into two categories; pattern-based encoders and optical interferometry-based encoders. Pattern-based encoders use a calibrated reference pattern strip to determine the displacement of the sensor head, but these too require close proximity between the moving stage and the reference frame in order to operate. Interferometric-based encoders do not have the same restriction and can be classified as non-contact, making them ideal for applications where space near the target surface is at a premium or where there is no suitable reference frame. In addition, optical interferometry can achieve very high operational measurement ranges whilst maintaining nanometre-level resolutions ${ }^{2}$. Typically, this comes at a cost of increased size and complexity of the interrogation system as well as various practical drawbacks such as the need, in general, to return the target object to a known reference position in case of a beam blockage.

However, optical interferometry remains an attractive technique for many high-precision applications, indeed over the years numerous variations on standard interferometric setups have been developed for metrological purposes in single dimensions ${ }^{3}$. When extended to more than one spatial dimension however, optical interferometric encoders typically require multiple sensing heads or optical access ports ${ }^{4,5}$. Alternative single optical access systems for multiple dimensions ${ }^{6-}$ ${ }^{9}$ have also been developed, often based on the use of optical gratings, but these frequently require complicated optical setups, increasing cost and sensitivity to environmental noise, and reducing fidelity. This current lack of low-complexity interferometric techniques severely limits the flexibility and versatility of optical interferometry over other encoding techniques for multi-dimensional measurements.

*t.kissinger@cranfield.ac.uk 
In this work, using our range-resolved interferometry (RRI) technique ${ }^{10}$ to measure interferometric phase changes at multiple locations along a single optical beam path, we demonstrate how displacement measurements along multiple dimensions can be made simultaneously using a very simple optical setup. Utilising optical frequency modulation resulting from sinusoidal injection current modulation of a simple continuous-wave laser diode, multiple interferometers can be evaluated using a single laser source and photodetector. RRI examines the superimposed interference pattern created by the multiplexing of all constituent interferometers within the beam path and separates their signals based on their optical path differences (OPD). In addition to fibre-optic strain ${ }^{11}$ and shape sensing ${ }^{12}$, we have previously applied RRI to multiplesurface laser vibrometry ${ }^{13}$, which allows movements of multiple semi-transparent surfaces along a single optical beam to be interrogated simultaneously for advanced vibration analysis. In this paper, we present a proof-of-concept experiment as a first demonstration of this principle for use as a multidimensional encoder, and present simultaneous displacement measurements in two dimensions of various stage movements. It is thought that the simplicity and space-effectiveness of this setup offers an attractive alternative to existing methods and could lead to new developments for its use as compact yet high resolution multi-dimensional remote optical encoders.

\section{EXPERIMENT}

In order to demonstrate this concept, the optical setup shown in Fig. 1(a) is used. Two planar aluminium mirrors are mounted on orthogonal faces of a Thorlabs $60 \mathrm{~mm}$ cage cube within stackable lens tubes. On an opposing side face of the cube, a fixed focus fibre collimator, comprising the single optical access port used in this setup, is mounted within a kinematic mount. Within the cage cube, a Physik Instrumente Nanocube stage (P-611.3) with a travel range of $\pm 50 \mu \mathrm{m}$ in three Cartesian dimensions is centrally positioned on top of a 2-axis goniometer and a rotation mount which allow for

(a)

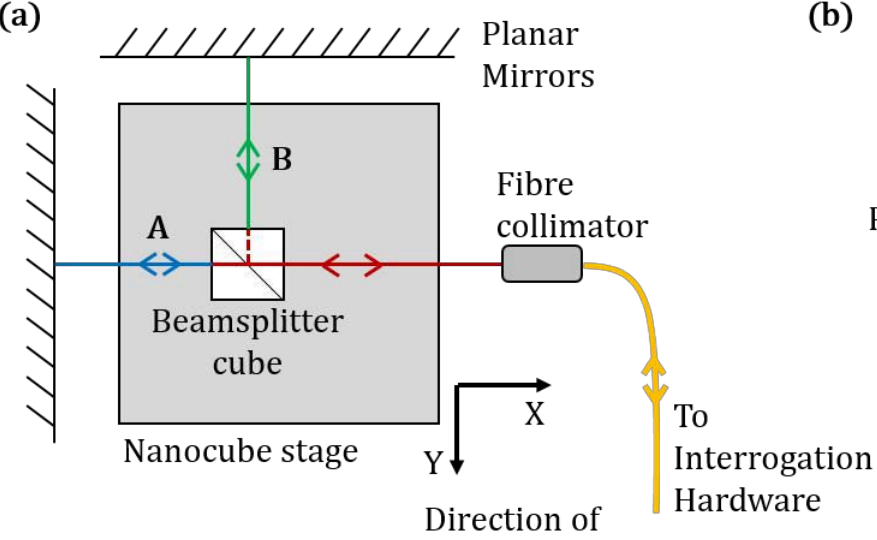

(c)

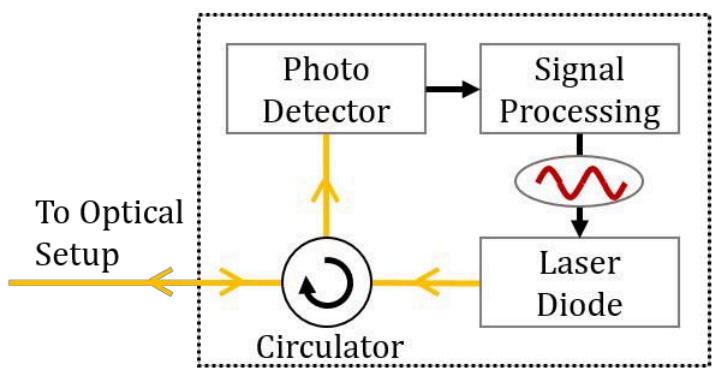

(b)

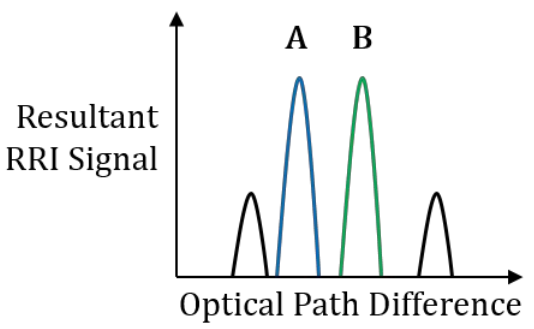

(d)

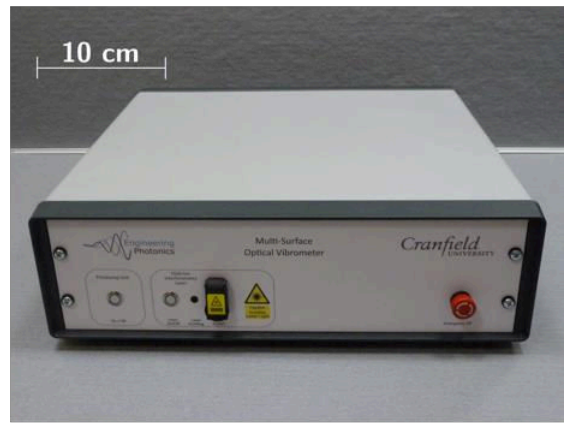

Figure 1. (a) shows a schematic of the optical setup used in this experiment. The blue and green optical paths mark the interferometers of interest, $\mathrm{A}$ and $\mathrm{B}$, formed between each mirror and the respective beam splitter end face. (b) then illustrates the expected RRI range view for this setup, where the signals from the desired interferometers A and B do not overlap with those peaks, marked in black, that are caused by secondary reflections in the system. The interrogation hardware is shown in (c), consisting of a photo detector, the digital signal processing hardware which also modulates a single continuous-wave laser diode, and a fibre-optic circulator. The fully enclosed interrogation unit is shown in (d). 
adjustment of the stage such that it is aligned with the axes of the cage cube. The stage utilises strain gauge sensors to feedback its position to the stage controller allowing for controlled, high precision movements. On the surface of the stage, a beamsplitter cube of $10 \mathrm{~mm}$ cube length with uncoated end faces is mounted along the optical axis of the collimated beam. The reflections from the two mirrors and the Fresnel reflection of the beamsplitter surface closest to the respective mirror then form the desired interferometers A and B, where the physical length of the interferometers A and B is chosen to differ by $7.5 \mathrm{~mm}$. Fig. 1(b) then shows an illustration of the resultant RRI range view that plots the return signal strength as a function of OPD. In this proof-of-concept device, the mirrors and beamsplitter are positioned such that the OPDs of the desired interferometers A and B are unique to the OPDs of all other constituent interferometers including those formed by secondary reflections, where any overlap of the OPDs of the desired interferometers with those caused by secondary reflections would cause cyclic errors in the measurements.

The RRI interrogation setup is drawn in Fig. 1(c) where the interrogation unit is based on a simple optical setup comprising of a diode laser source (Eblana EP1512-DM-B, wavelength: $\sim 1521 \mathrm{~nm}$, power: $\sim 5 \mathrm{~mW}$, linewidth: $\sim 1 \mathrm{MHz}$ ) that has its emission wavelength sinusoidally modulated at a frequency of $49 \mathrm{kHz}$ by modulating the laser injection current, resulting in a wavelength modulation amplitude of $\sim \pm 0.2 \mathrm{~nm}$. The interrogation system is capable of resolving interferometric bandwidths up to $21 \mathrm{kHz}$ for a total of 24 range channels. In Fig. 1(c) the light of the laser diode is guided by a fibre-optic circulator, via a single-mode fibre lead, to a collimator where it traverses the setup shown in Fig. 1(a). The returning light is guided by the circulator to an InGaAs photo detector and the resulting interferometric signals are demodulated ${ }^{10}$ in real time by field programmable gate array (FPGA)-based signal processing hardware before being transferred to a PC. Fig. 1(d) then shows a picture of the fully-enclosed interrogation unit that houses the complete measurement system including a PC.

\section{RESULTS AND DISCUSSION}

In order to demonstrate the measurement capabilities of this concept, two-dimensional displacement measurements over a variety of stage movements were conducted and recorded with an interferometric quadrature bandwidth of $21 \mathrm{kHz}$. In the first experiment, starting from a central position, the stage was moved to a fixed offset in the X-direction, then swept to the same but negative offset in the X-direction before being returned to the central position. This is then repeated in the Ydirection shortly after.

In Fig. 2(a), simultaneous measurements of $\mathrm{X}$ and $\mathrm{Y}$ displacements are shown for stage motions of $\pm 50 \mu \mathrm{m}$. These are shown for a stage motion in $\mathrm{X}$ to $50 \mu \mathrm{m}$, followed by a stage movement in $\mathrm{X}$ to $-50 \mu \mathrm{m}$ at a velocity of $10 \mu \mathrm{m} / \mathrm{s}$, before the stage is returned to the central position. This is then repeated in Y after a short delay. Fig. 2(b) shows the same data as Fig. 2(a) but viewing a smaller range around the zero-displacement position in order to better see any parasitic motion from the non-moving axis during each motion. Fig. 2(c) shows the displacements measured in $\mathrm{X}$ and $\mathrm{Y}$ due to a similar motion as above, but with a range of \pm 5 um and with velocities of $1 \mu \mathrm{m} / \mathrm{s}$. Fig. $2(\mathrm{~d})$ then again shows a closer range around the zerodisplacement axis for the same data as in Fig. 2(b). With a range of $\pm 0.5 \mu \mathrm{m}$ and velocities of $0.1 \mu \mathrm{m} / \mathrm{s}$, the same motion patterns are repeated as above, and the $\mathrm{X}$ and $\mathrm{Y}$ displacement measurements are shown in Fig.2(e), and with Fig.2(f) showing a yet closer view of the zero-displacement axis.

In addition to demonstrating the measurement of stage displacement, this experiment can also be used to estimate the typical noise levels achievable with this interrogation system. By analysis of the stationary part of the signal, between the two motion patterns, typical noise standard deviations of $3 \mathrm{~nm}$, or noise densities of $0.02 \mathrm{~nm} / \sqrt{ } \mathrm{Hz}$, are obtained over an interferometric quadrature bandwidth of $21 \mathrm{kHz}$.

The results in Fig. 2 demonstrate that independent measurements of movement in the two orthogonal stage directions can be simultaneously performed with this concept. Closer examination of the displacement measurement for the dimension orthogonal to the intended motion of the stage, shown in Figs. 2(b), (d) and (f) shows a slight parasitic movement from the expected zero position where the expectation is that the measured displacement would ideally remain static. This is believed to be a real effect and not an artefact of the measurement, as across Figs. 2(b), (d) and (f) this effect can be seen to be approximately proportional to the total displacement of the stage. This parasitic displacement orthogonal to the desired movement axis could be caused through misalignment of the stage motion to the optical axis of the incident beam, through non-planarity of the mirrors used or through genuine motion imperfections of the stage. 

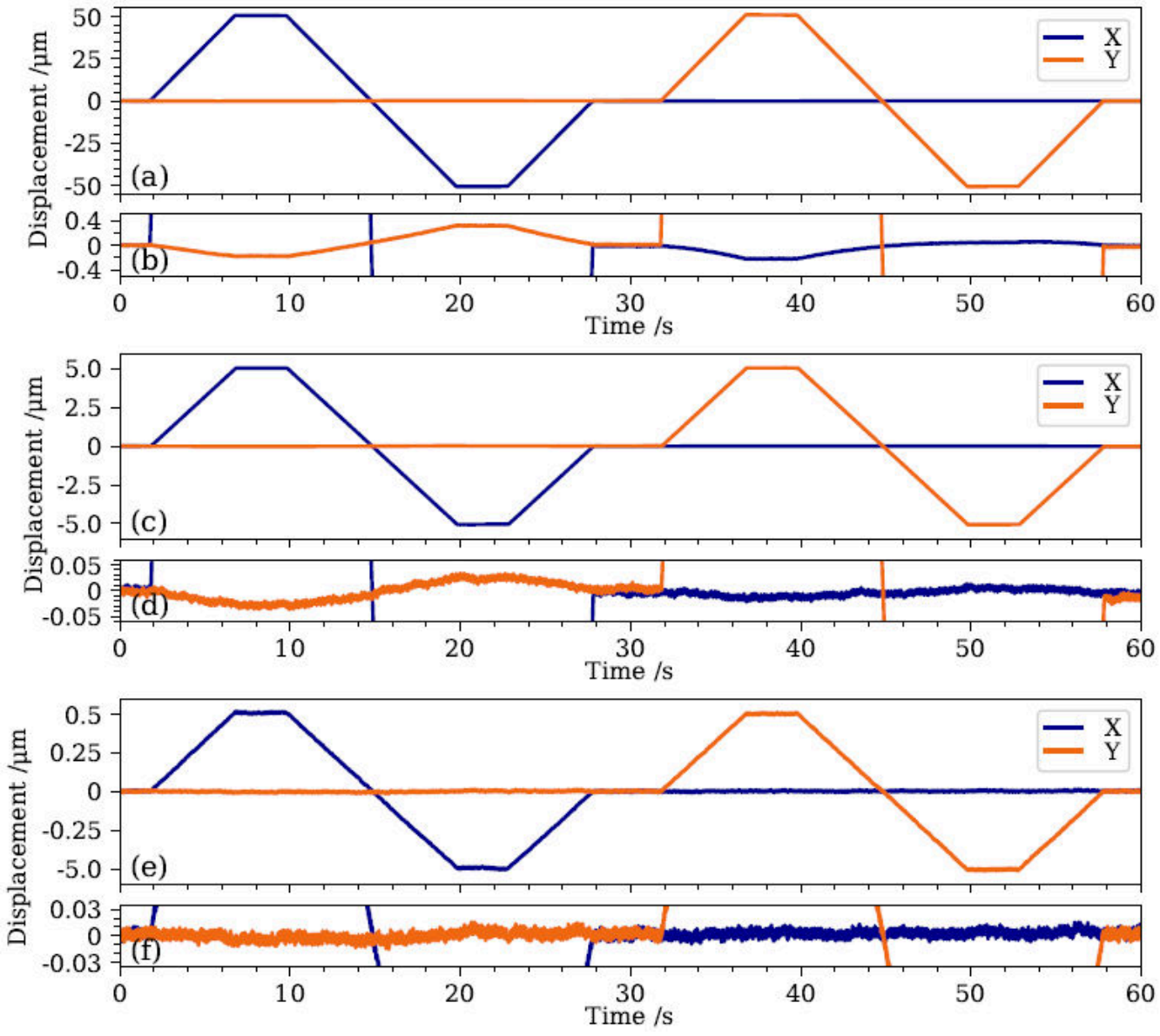

Figure 2. Simultaneous measurements of $\mathrm{X}$ and $\mathrm{Y}$ displacements for motion in the $\mathrm{X}$-direction of the stage followed by a duplicate motion in the $\mathrm{Y}$-direction. (a) shows a movement across the total range of the stage, \pm 50 $\mu \mathrm{m}$ at a speed of $10 \mu \mathrm{m} / \mathrm{s}$, while (b) shows a closer view of the same results over a smaller displacement range around the zero-displacement position. (c) and (d) plot the results in a similar fashion to (a) and (b) but for a smaller stage movement across $\pm 5 \mu \mathrm{m}$ at velocities of $1 \mu \mathrm{m} / \mathrm{s}$. (e) and (f) then show similar plots for $\pm 0.5 \mu \mathrm{m}$ movements at velocities of $0.1 \mu \mathrm{m} / \mathrm{s}$.

The parasitic displacement orthogonal to the desired motion direction is further analysed by plotting the $\mathrm{X}$ and $\mathrm{Y}$ displacement signals across the stage operating range directly against each other in Fig. 3. Here, the plotted data is taken from the same data set that was previously shown in Fig. 2(a), using the interval between 0 and $30 \mathrm{~s}$ for the $\mathrm{X}$-motion data and the 30 to $60 \mathrm{~s}$ interval for the Y-motion data.

Fig. 3(a) directly plot the results for the two datasets for a desired $\mathrm{X}$ and $\mathrm{Y}$ motion. Fig. 3(b) then enlarges the measurement for the $\mathrm{Y}$ motion around the zero $\mathrm{X}$ displacement axis while Fig. 3(c) plots the $\mathrm{X}$ motion measurement around the zero $\mathrm{Y}$ motion axis. As expected, in the directions orthogonal to the intended motion of the stage there is a parasitic motion component visible in both Figs. 3(b) and (c). For the X-motion shown in Fig. 3(c) this parasitic motion component is mostly linear but does exhibit a small hysteresis, which is likely to be a real effect originating from the Piezoelectic stage used. For the Y-motion in Fig. 3(b), the hysteresis is also present but smaller in magnitude, with the parasitic motion component being less linear than in Fig. 3(b). The occurrence of the hysteresis for the bi-directional motion is a phenomena generally associated with Piezoelectic stages and it is therefore expected that this measurement shows a real effect and is not a measurement artefact, highlighting that the high-quality two-dimensional measurements achievable with this approach can be used to resolve sub- $\mu \mathrm{m}$ irregularities of the stage motions. 

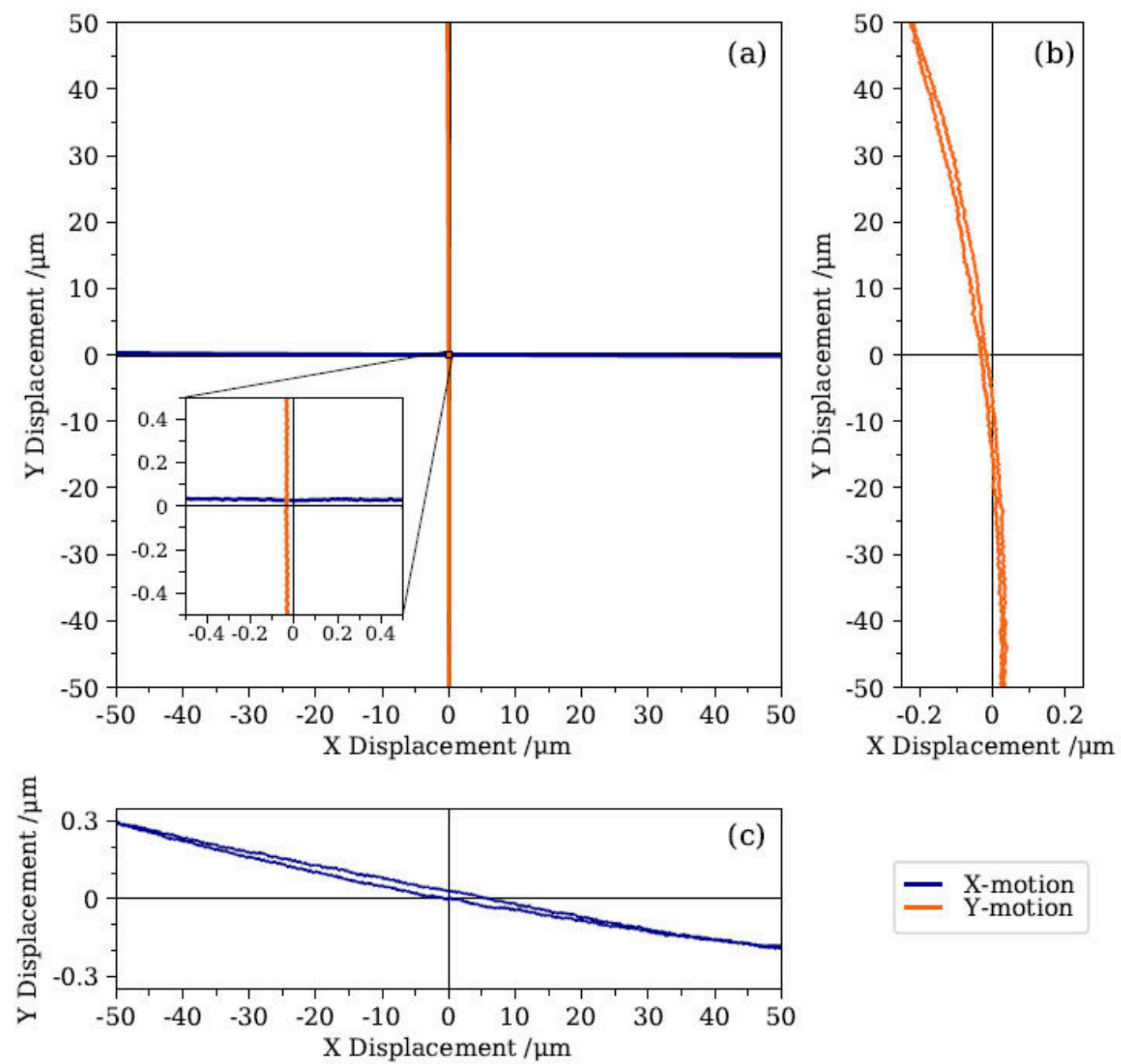

Figure 3. (a) shows the direct $\mathrm{X}$ against $\mathrm{Y}$ plots of the measured displacements for stage movements in both $\mathrm{X}$ and $\mathrm{Y}$ directions over the entire $\pm 50 \mu \mathrm{m}$ operating range of the stage. These correspond to the $-50 \mu \mathrm{m}$ to $50 \mu \mathrm{m}$ motions shown in Fig. 2(a). (b) shows the same results on a tighter range around the zero $\mathrm{X}$ displacement line, with only the results corresponding to the Y-motion plotted. (c) shows the same results as in (a) on a tighter range around the zero $\mathrm{Y}$ displacement line with only the results corresponding to the $\mathrm{X}$ motion plotted.

To further investigate the measurement capabilities of the proof-of-concept sensor for simultaneous motion in multiple motion directions, a second experiment was undertaken. In this experiment, the stage was moved in a circular path with a radius equal to $50 \mu \mathrm{m}$. This corresponds to a sinusoidal motion in the X-direction with an amplitude of $50 \mu \mathrm{m}$ and a sinusoidal motion in the Y-direction with the same amplitude and a phase offset of $\pi / 2$. This results in a circular motion over the entire range of motion of the stage in the X-Y plane. The results of this experiment are shown in Fig. 4. As expected from circular motion, the individual $\mathrm{X}$ and $\mathrm{Y}$ displacement components shown in Fig. 4(b) both exhibit a sinusoidal pattern with a phase difference of $\pi / 2$ between them. The results shown in Fig. 4 clearly demonstrate that highquality simultaneous measurements in two motion directions are possible using the proposed approach. 

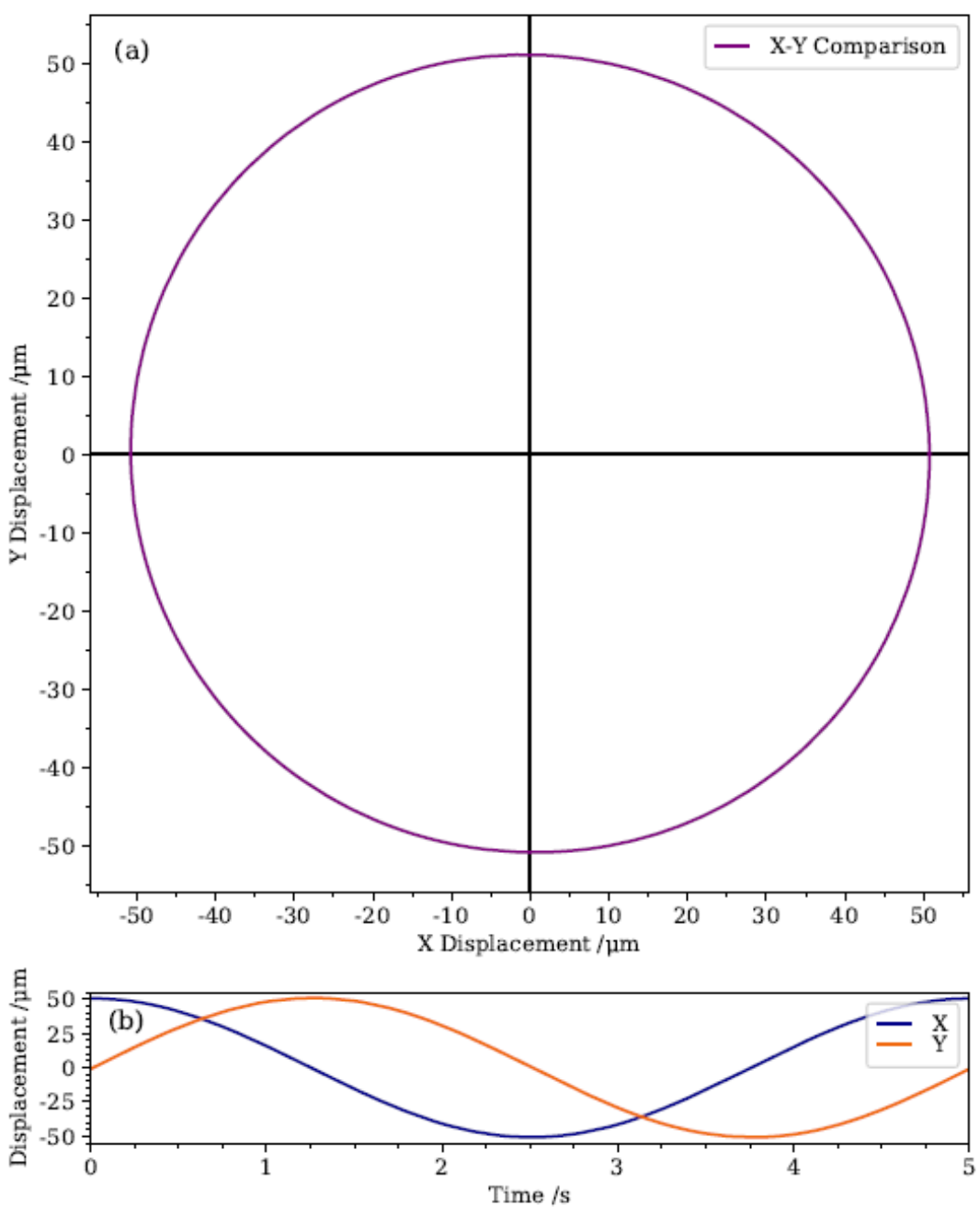

Figure 4. (a) shows displacement measurements recorded over a circular motion in the X-Y plane of the stage. This motion utilised the entire $\pm 50 \mu \mathrm{m}$ travel range of the stage. Figure (b) shows the individual $\mathrm{X}$ and $\mathrm{Y}$ displacement measurements against time for one circular movement of the stage with a period of 5 seconds.

\section{CONCLUSION}

Using a proof-of-concept experiment we have demonstrated that, using a range-resolved interferometric approach, simultaneous displacement measurements for two dimensions can be performed within a simple optical setup and using only a single optical axis port. This technique utilises a single laser diode and photodetector making it cost-effective and maintaining the ability for remote measurements that is a major benefit of interferometric encoders compared to other position encoder techniques The presented measurements over a nominal stage working range of $\pm 50 \mu \mathrm{m}$ clearly demonstrate independent, simultaneous displacement measurements of two dimensions with typical noise densities of 0.02 $\mathrm{nm} / \sqrt{ } \mathrm{Hz}$ over a $21 \mathrm{kHz}$ bandwidth, with this resolution comparable or exceeding existing optical encoders. A future, more advanced implementation of this approach, including laser wavelength control to allow traceable measurements, could result in a high-accuracy yet compact optical encoder capable of remote multi-dimensional displacement measurements. However, even the demonstrated proof-of-principle experiment alone could be easily adapted for other applications in areas such as manufacturing or industrial applications that would benefit from an optically simple and compact approach to dimensional metrology. 


\section{ACKNOWLEDGEMENTS}

The authors acknowledge the support of the Royal Society UK via Paul Instrument Fund grant PI150046 and of the Engineering and Physical Sciences Research Council (EPSRC) UK via grants EP/M020401/1 and EP/N002520/1 and via EPSRC Doctoral Training Partnership EP/N509450/1.

\section{REFERENCES}

[1] Fleming, A. J., “A review of nanometer resolution position sensors: Operation and performance," Sensors and Actuators, A: Physical 190, 106-126 (2013).

[2] Gao, W., Kim, S. W., Bosse, H., Haitjema, H., Chen, Y. L., Lu, X. D., Knapp, W., Weckenmann, A., Estler, W. T., and Kunzmann, H., "Measurement technologies for precision positioning," CIRP Annals - Manufacturing Technology 64(2), 773-796 (2015).

[3] Bobroh, N., "Recent advances in displacement measuring interferometry," Measurement Science and Technology 4(9), 907 (1993)

[4] Pan, S.-W., Hsieh, H.-L., and Wang, W.-C., "6-DOF displacement and angle measurements using heterodyne laser encoder," Instrumentation, Metrology, and Standards for Nanomanufacturing, Optics, and Semiconductors VII 8819(43), 881909 (2013).

[5] Chung, Y. C., Fan, K. C., and Lee, B. C., "Development of a novel planar encoder for 2D displacement measurement in nanometer resolution and accuracy," Proceedings of the World Congress on Intelligent Control and Automation (WCICA), 449-453 (2011).

[6] Hsu, C.-C., Chen, H., Chiang, C.-W., and Chang, Y.-W., "Dual displacement resolution encoder by integrating single holographic grating sensor and heterodyne interferometry," Optics Express 25(24), 30189 (2017).

[7] Kimura, A., Gao, W., Arai, Y., and Lijiang, Z., "Design and construction of a two-degree-of-freedom linear encoder for nanometric measurement of stage position and straightness," Precision Engineering 34(1), 145-155 (2010).

[8] Wu, C.-C., Chen, Y.-Z., and Liao, C.-H., “Common-path laser planar encoder,” Optics Express 21(16), 18872 (2013)

[9] Lee, C., Kim, G. H., and Lee, S. K., "Design and construction of a single unit multi-function optical encoder for a six-degree-of-freedom motion error measurement in an ultraprecision linear stage," Measurement Science and Technology 22(10) (2011).

[10] Kissinger, T., Charrett, T. O.H. and Tatam, R. P., "Range-resolved interferometric signal processing using sinusoidal optical frequency modulation," Optics Express 23(7), 9415 (2015).

[11] Kissinger, T., Correia, R., Charrett, T.O.H., James, S.W. and Tatam, R.P., "Fiber segment interferometry for dynamic strain measurements," Journal of Lightwave Technology 34(19), 4620-4626 (2016).

[12] Kissinger, T., Chehura, E., Staines, S.E., James, S.W. and Tatam, R.P., "Dynamic fiber-optic shape sensing using fiber segment interferometry," Journal of Lightwave Technology 36(4), 917-925 (2018).

[13] Kissinger, T., Charrett, T.O.H, James, S.W., Adams, A., Twin, A. and Tatam, R.P., "Simultaneous laser vibrometry on multiple surfaces with a single beam system using range-resolved interferometry," Proc. SPIE 9525, 952520 (2015). 
Two-dimensional remote interferometric stage encoder through a single access port using range-resolved interferometry

Wiseman, Kieran B.

SPIE

Wiseman KB, Kissinger T, Tatam RP. (2019) Two-dimensional remote interferometric stage encoder through a single access port using range-resolved interferometry. In: SPIE Optical Metrology: Optical Measurement Systems for Industrial Inspection XI, 24-27 June 2019, Munich, Germany https://doi.org/10.1117/12.2525596

Downloaded from Cranfield Library Services E-Repository 